\title{
The Effect of Temperature on the Gasification Process
}

\author{
Marek Baláśs ${ }^{1}$, Martin Lisý $^{1}$, Ota Štelcl ${ }^{1}$ \\ ${ }^{1}$ Brno University of Technology, Faculty of Mechanical Engineering, Energy Institute, Technická 2,61669 Brno, Czech \\ Republic
}

Correspondence to: balas.m@fme.vutbr.cz

\begin{abstract}
Gasification is a technology that uses fuel to produce power and heat. This technology is also suitable for biomass conversion. Biomass is a renewable energy source that is being developed to diversify the energy mix, so that the Czech Republic can reduce its dependence on fossil fuels and on raw materials for energy imported from abroad.

During gasification, biomass is converted into a gas that can then be burned in a gas burner, with all the advantages of gas combustion. Alternatively, it can be used in internal combustion engines. The main task during gasification is to achieve maximum purity and maximum calorific value of the gas. The main factors are the type of gasifier, the gasification medium, biomass quality and, last but not least, the gasification mode itself.

This paper describes experiments that investigate the effect of temperature and pressure on gas composition and low calorific value. The experiments were performed in an atmospheric gasifier in the laboratories of the Energy Institute at the Faculty of Mechanical Engineering, Brno University of Technology.
\end{abstract}

Keywords: biomass, gasification, temperature effect.

\section{Introduction}

Biomass is one of the most significant renewable energy sources worldwide. Biomass technology has many advantages, e.g. there is a comparatively low negative impact on the environment, and it can be grown on surplus agricultural land that is not suitable for, or is not required for, food production purposes.

In recent times, renewable energy sources have covered almost $4 \%$ of power production in the Czech Republic, and biomass is a major contributor to these sources.

There are various ways in which biomass can be used for heat and power production, ranging from oil esterification, biogas production and biogas utilization to thermal processes such as pyrolysis, combustion and gasification. At the present time, direct combustion is the most widely used method for producing power from biomass. It is the oldest method, with well-mastered technologies. However, low-capacity processing lines are mostly suitable for heat production from biomass, and not for more desirable power production.

Fuel gasification, with subsequent utilization of the generated gas in a cogeneration unit, is another technology for producing power from biomass. Gasification is a relatively new method that offers many advantages. Increased efficiency of energy utilization from biomass, especially power production, is a major advantage of the gasification process. Combustion of the gas that is produced is a more easily controlled process than combustion of solid biomass. Gasification therefore decreases the production of harmful emissions. Higher power production efficiency is achieved using gas in gas turbines and steam-gas cycles. Gasification also achieves lower heat loss and better energy production than biogas combustion [1].

Gasification is the thermo-chemical conversion of organic mass with a limited oxygen supply into a lower calorific value gas $\left(4-15 \mathrm{MJ} / \mathrm{m}_{n}^{3}\right)$ whose main components include: $\mathrm{CO}, \mathrm{CO}_{2}, \mathrm{H}_{2}, \mathrm{CH}_{4}$, more complex hydrocarbons, $\mathrm{N}_{2}$ and pollutants. The operating temperatures are rather high, commonly from 750 up to $1000^{\circ} \mathrm{C}$. The gas that is produced is then combusted in a boiler or in combustion engines (and/or combustion turbines). The use of air as the gasification medium results in a low calorific value of the gas $\left(4-7 \mathrm{MJ} / \mathrm{m}_{n}^{3}\right)$ due to dilution of the gas with nitrogen (more than $50 \%$ ). The use of mixtures of air and oxygen and/or steam as the gasification medium produces gas of lower calorific value, ranging from $10-15 \mathrm{MJ} / \mathrm{m}_{n}^{3}[2]$. Heat for endothermic reactions is most commonly produced by partial oxidation of the gasified material (air or oxygen as the gasification medium), or is supplied from external sources. The main purpose of the gasification process is to transform as much energy as possible from fuel into gas [3].

The most monitored characteristics of the gas that is produced include quality (lower calorific value, composition), the amount produced by means of gasification, and also the amount of pollutants in the gas, 
and their composition. Our study focuses on the effect of temperature and pressure on the quality and composition of the gas that is produced.

\section{Methodology for measurements at the biofluid 100 gasification fluid generator}

The study was performed at the Biofluid 100 stand (see Figure 1), which is equipped with a stationary fluidized bed.

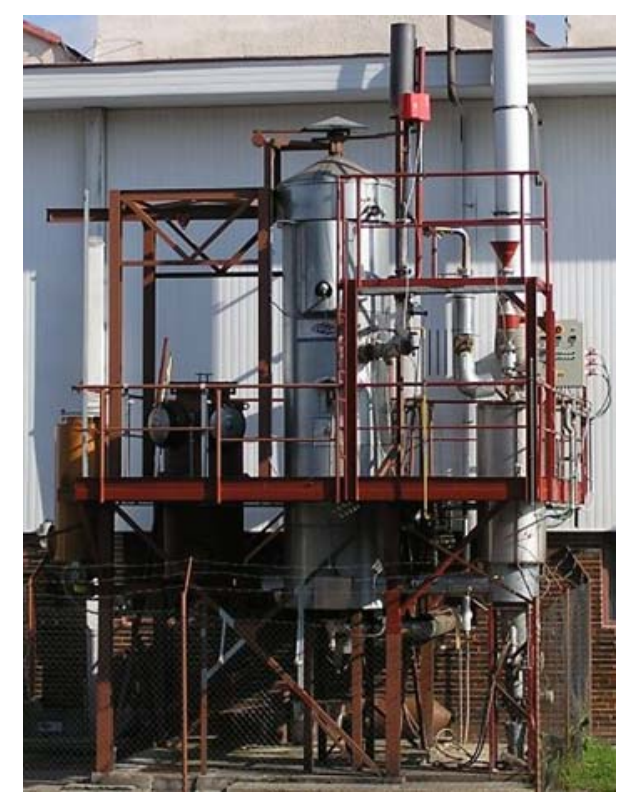

Figure 1: Biofluid 100 experimental equipment

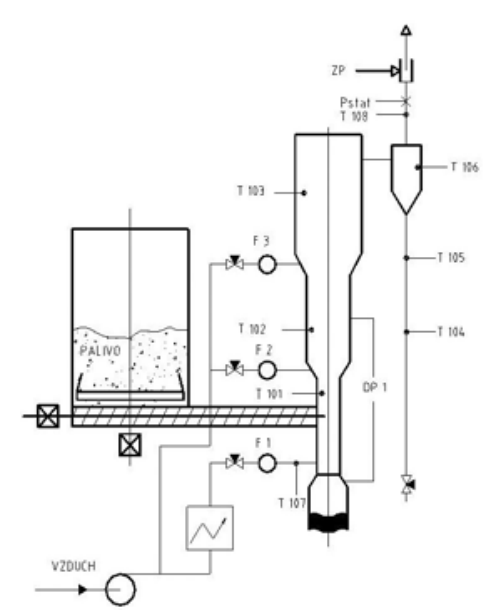

Figure 2: Scheme of the Biofluid gasifier
A simplified scheme of the experimental equipment is presented in Figure 2. Fuel is supplied from a fuel storage tank equipped with a shovel, and is introduced via a dosing screw with a frequency converter into the reactor. The primary supply of blower compressed air is led into the reactor under the bed, and secondary and tertiary supplies are located at two high-rise levels. The energogas that is produced is stripped of its solid particulate matter in the cyclone. The output gas is combusted in a burner equipped with a stabilization burner for natural gas and an individual air supply. The ash from the reactor can be removed from the tank located beneath the bed. The power-based heater for primary air supply is placed behind the blower to enable the impact of air preheating to be monitored. In recent years, filters for a study of the efficiency of various gas cleaning methods have been attached to basic part of stand.

Reactor parameters:

- Capacity (in produced gas) $100 \mathrm{~kW}_{t}$

- Fuel demand (consumption, requirement) $150 \mathrm{~kW}_{t}$

- Wood consumption $40 \mathrm{~kg} \cdot \mathrm{h}^{-1}$

- Air flow rate $50 \mathrm{~m}_{n}^{3} \cdot \mathrm{h}^{-1}$

The basic fluid generator operation characteristics are described below:

- Operation of the fluid generator - after ignition, the fluid generator is operated in combustion mode, so that its heating is quickit heats up quickly. After the required gasification temperatures are achieved, secondary and tertiary air is supplied to the generator, and thus the gas is immediately combusted and consequently heats up the generator. The air supplies are then shut off, and the generator is introduced into stable mode for a specific and preset gasification temperature. A stable mode is achieved when the amount of fuel that is dosed remains unaltered, the amount of gasified air is even, and the temperature swings in middle section of the gasification generator are stable within the narrow range specified by the gasification temperature.

- Data entry for the gasification process the monitored data is continuously recorded by a computer at time intervals of 10 seconds for each measurement. The following values are monitored:

- The frequency of the converter of the dosing screw, for determining the mass flow rate;

- The temperature in various parts of equipment, which is measured by thermocouples; the positions of the thermocouples are given in detail in the scheme in Figure 2. There are 3 thermocouples along the top of the generator, 1 thermocouple in the cyclone and 2 thermocouples in the semi-coke pipe. 
One thermocouple is in the output gas pipe. One thermocouple measures the temperature of the primary air supply.

- The pressure difference between the upper and lower sections of the fluid generator (fluid bed);

- The pressure difference at the orifice plate, for determining the gas flow rate;

- The pressure of the generated gas, at the generator outlet and at the fuel storage tank.

Other values, e.g. temperature and air moisture, primary air flow rate and primary air temperature, have to be recorded manually.

\section{Course of the experiments}

The main purpose of the experiments was to determine the dependency of gas quality on pressure and temperature changes in the gasifier. The quality of the gas was assessed by analyzing its composition and its lower calorific value. A device based on infrared spectrometry monitored the gas composition ( $\mathrm{CO}$ and $\mathrm{H}_{2}$ components) online after the stand had stabilized. Gas was also sampled into a test-glass at regular intervals. These gas samples were later analyzed in a gas chromatograph $\left(\mathrm{H}_{2}, \mathrm{CO}, \mathrm{CO}_{2}, \mathrm{CH}_{4}\right.$ components). Further required parameters for the samples were set later.

\section{Results and discussion}

The dependency of the gas composition on the temperature and pressure in the gasification generator was experimentally monitored. No dependency of gas composition on fluid bed temperatures ( $\mathrm{T} 101$ and $\mathrm{T}$ 102) or on other values was proved. On the contrary, the experimental results showed that the temperature in the freeboard section ( $\mathrm{T}$ 103), where the chemical balance reactions take place, is the key temperature for gas composition (see Figure 3 through Figure10). The charts show that an increase in temperature results in an increase in the proportion of hydrogen and carbon monoxide, and a decrease in the proportion of carbon dioxide and methane. This is due to the decreasing speed of the methanizing reactions, and the higher probability of reactions of water gas $\left(\mathrm{C}+\mathrm{H}_{2} \mathrm{O} \rightarrow \mathrm{CO}+\mathrm{H}_{2}\right)$. The available equipment places limitations on analyses of dependency on pressure. While the temperature can be set within a range of $100{ }^{\circ} \mathrm{C}$, the pressure can be regulated only within the range from ca. 2.5 to $19 \mathrm{kPa}$. This is a rather narrow range. However, an increased proportion of methane is seen to be dependent on pressure, i.e. this dependency contrasts with the temperature trends.

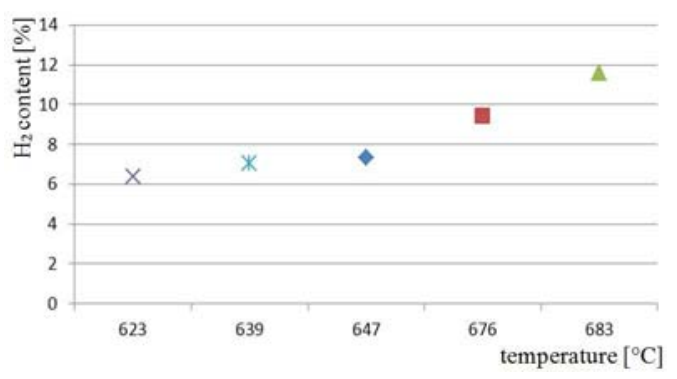

Figure 3: Chart of the dependency of $\mathrm{H}_{2}$ content on T 103 temperature

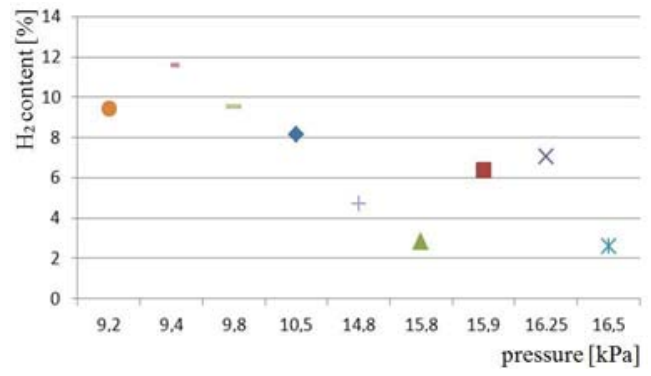

Figure 4: Chart of the dependency of $\mathrm{H}_{2}$ content on pressure

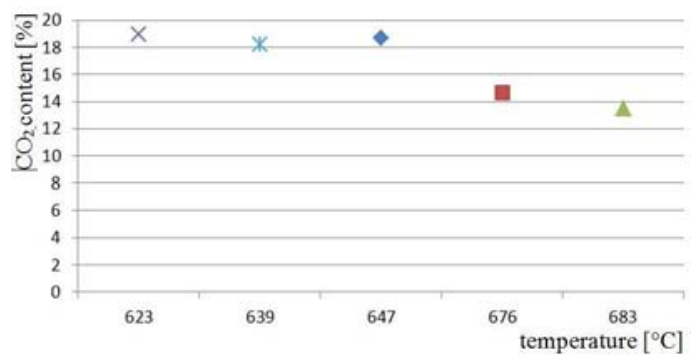

Figure 5: Chart of the dependency of $\mathrm{CO}_{2}$ content on T 103 temperature

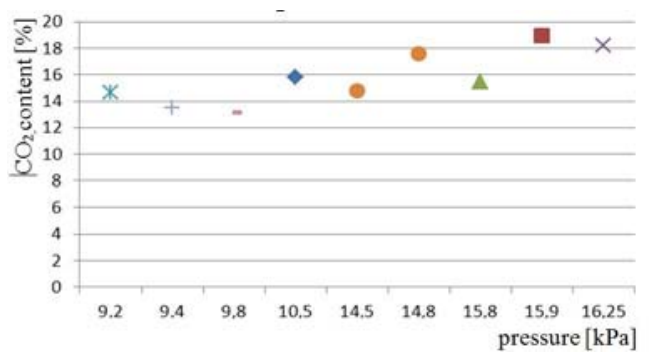

Figure 6: Chart of the dependency of $\mathrm{CO}_{2}$ content on pressure 


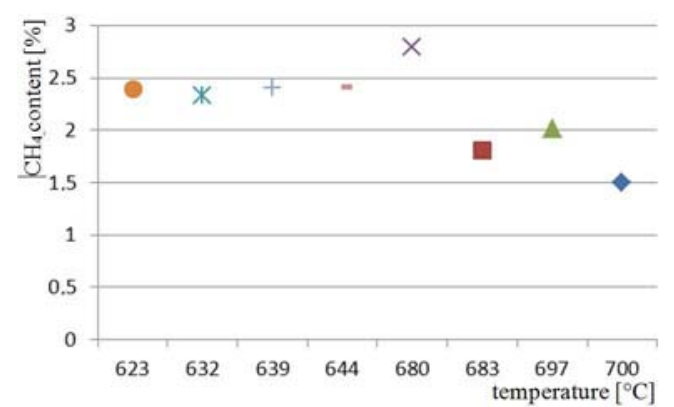

Figure 7: Chart of the dependency of $\mathrm{CH}_{4}$ content on T 103 temperature

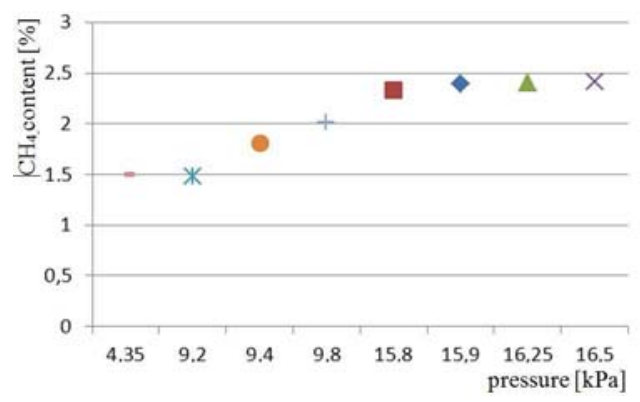

Figure 8: Chart of the dependency of $\mathrm{CH}_{4}$ content on pressure

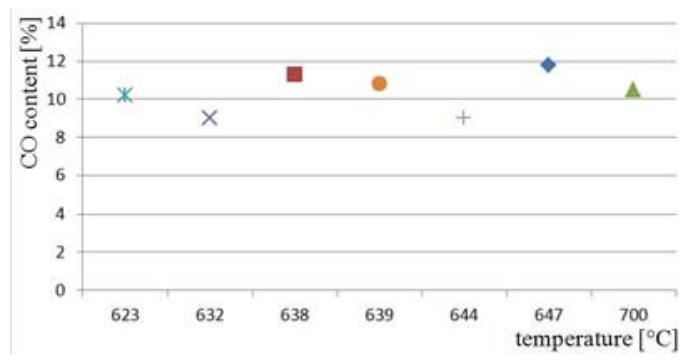

Figure 9: Chart of the dependency of CO content on T 103 temperature

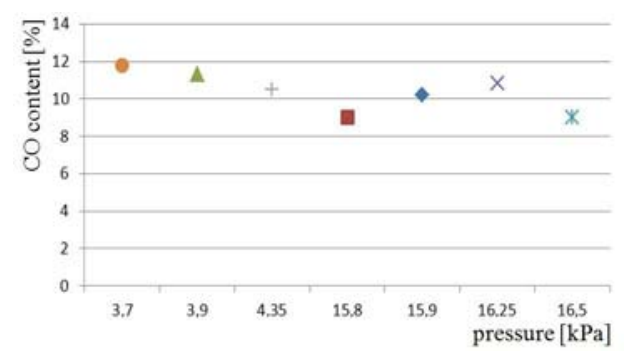

Figure 10: Chart of the dependency of CO content on pressure

Subsequent analyses of the generated gas focused on its lower calorific value. As Figure 11 shows, an increase in temperature results in a slight decrease in the proportion of nitrogen in the gas. Thus, we see an increase in the proportion the combustible component in the gas, and therefore an increase in the lower calorific value of the gas (see Figure 13). The dependency on pressure again contrasts with the dependency on temperature (see Figures 12 and 14).

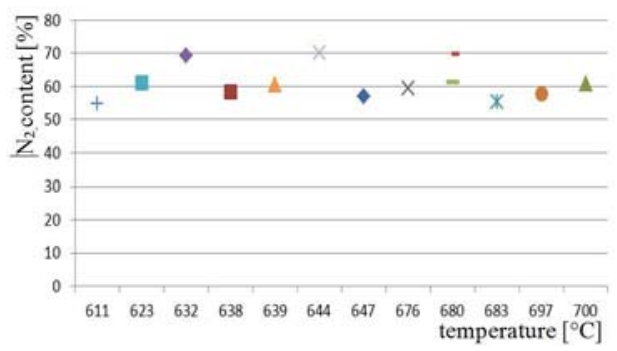

Figure 11: Chart of the dependency of $\mathrm{N}_{2}$ content on T 103 temperature

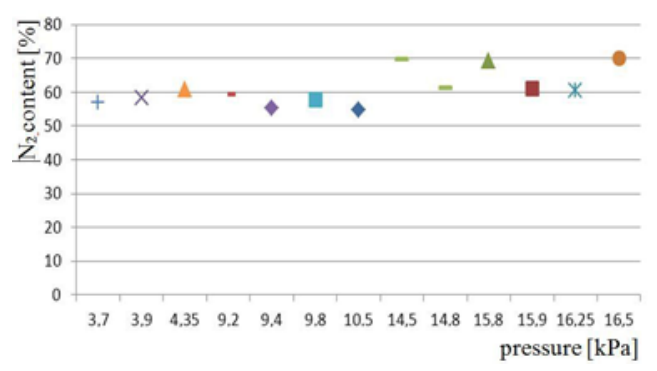

Figure 12: Chart of the dependency of $\mathrm{N}_{2}$ content on pressure

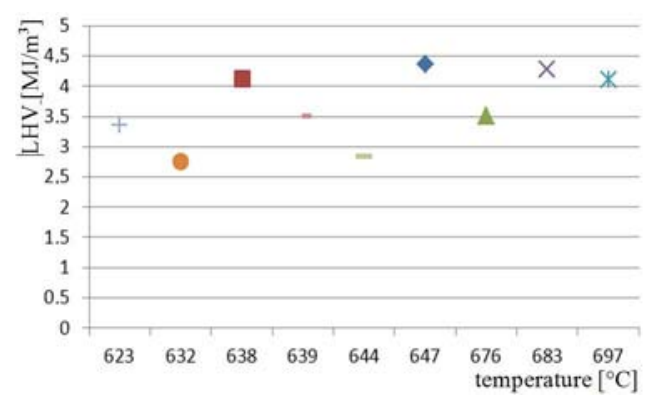

Figure 13: Chart of the dependency of lower calorific value on $\mathrm{T} 103$ temperature

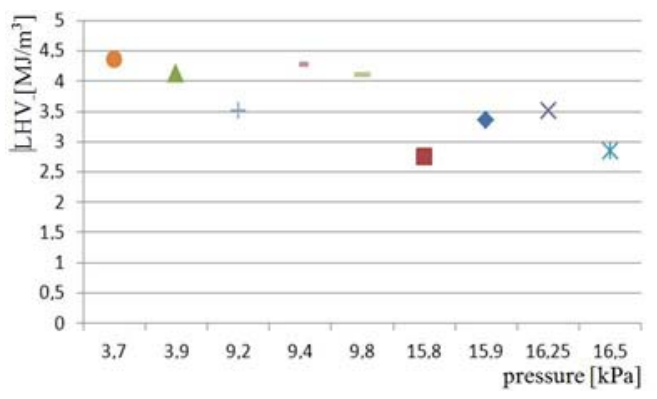

Figure 14: Chart of the dependency of lower calorific value on pressure

\section{Conclusion}

This paper has analyzed the effect of gasification temperature and pressure on the quality of the gas gen- 
erated from biomass. The results show that the temperature in the fluid bed of a gasification reactor has no effect whatsoever on the final composition of the gas. However, T 103, i.e. the temperature in freeboard, has a direct impact on the final composition of the gas. An increase in temperature results in an increase in the proportion of $\mathrm{CO}$ and $\mathrm{H}_{2}$, a decrease in the proportion of $\mathrm{CH}_{4}$ and $\mathrm{CO}_{2}$, and an increase in the lower calorific value of the gas. A change in pressure has the opposite effect.

\section{Acknowledgement}

Financial support from GAČR project No. $101 / 09 / 1464$ is gratefully acknowledged.

\section{References}

[1] Lisý, M., Baláš, M., Moskalik, J., Pospíšil, J.: Research into biomass and waste gasification in atmospheric fluidized bed, 2009, Proceedings of the 3rd WSEAS International Conference on Renewable Energy Sources, RES '09. ISBN 978-960-474-093-2.

[2] Baláš, M., Lisý, M.: Vliv vodní páry na proces zplyňování biomasy, Acta Metallurgica Slovaca, Vol. 11, No. 1, Košice, 2005. ISSN 1335-1532.

[3] Ochrana, L., Skála, Z., Dvořák, P., Kubíček, J., Najser, J.: Gasification of Solid Waste and Biomass, VGB PowerTech, 2004, Vol. 84, No. 6, p. 70-74. ISSN 1435-3199. 